ENFOQUES JURIDICOS

REVISTA MULTIDISCIPLINAR DEL CEDEGS

ISSN 26832070

Número 03

enero-junio 2021
Artículo: “El problema de la relación entre derecho y moral"

José Luis López Fuentes

DOI: https://doi.org/10.25009/ej.v0i3.2563

\title{
El problema de la relación entre derecho y moral
}

Recibido 15 noviembre 2020-Aceptado 15 febrero 2021

\author{
José Luis López Fuentes* \\ Xalapa-Equez. Veracruz, México \\ luis.jll@@gmail.com
}

RESUMEN: En el presente trabajo, con base en las teorías iusnaturalistas $y$ del positivismo jurídico, se busca ofrecer un breve acercamiento al desarrollo que han tenido a través del tiempo las tesis más importantes en torno al problema de la relación entre derecho y moral, hasta llegar a lo que actualmente es denominado antipositivismo jurídico, pues el objetivo de este documento es presentar un análisis y exposición de las aportaciones de esta corriente de pensamiento a la teoría jurídica contemporánea, para lo cual, se analizan las propuestas de Ronald Dworkin y Robert Alexy, en especial de la tesis de los principios, y su relevancia en la interpretación y aplicación de la ley.

Palabras clave: Iusnaturalismo, positivismo jurídico, antipositivismo jurídico, moral, tesis de los principios.
ABSTRAC: In this work, based on natural law theories and legal positivism, I seek to offer a brief approach to the development that the most important theses have had throughout time regarding the problem of the relationship between law and morality, arriving at what we now call legal antipositivism, the objective of this document is to present an analysis and exposition of the contributions of this current of thought to contemporary legal theory, for which the proposals of Ronald Dworkin and Robert Alexy are analyzed, specially the thesis of the principles, and its relevance in the interpretation and application of the law.

Keywords: Natural law theories, legal positivism, legal antipositivism, moral, thesis of principles.

SUMARIO: Introducción, 1. Contexto iusnaturalista. 2. Contexto iuspositivista. 3. Moderna teoría antipositivista. Consideraciones finales. Fuentes de consulta.

* Licenciado en Derecho por la Universidad Veracruzana.

pp. $65-80$

CEVES 
ENFOQUES JURIDICOS

REVISTA MULTIDISCIPLINAR DEL CEDECS

ISSN 26832070

Número 03

enero-junio 2021
Artículo: “El problema de la relación entre derecho y moral"

José Luis López Fuentes

DOI: https://doi.org/10.25009/ej.v0i3.2563

\section{Introducción}

En términos generales el problema de la relación entre derecho y moral ha sido abordado a lo largo de la historia desde dos vertientes de pensamiento (con diversos matices). En primer lugar, se encuentran las teorías elaboradas desde el derecho natural o iusnaturalismo, las cuales se caracterizan por postular que el derecho es válido y obligatorio no por su fuente formal de origen, sino por la justicia de su contenido. En segundo lugar, se encuentran las teorías del positivismo jurídico o iuspositivismo, las que se distinguen porque buscan teorizar sobre el derecho de un modo general y descriptivo. Se habla de generalidad en el sentido de intentar estudiar al derecho en su totalidad y no solo a un sistema jurídico en particular, por otro lado, uno de los sentidos en que estas teorías buscan ser descriptivas, se refiere a que pretenden ser neutrales y no comprometerse con ninguna opción moral o política.

El objetivo de este trabajo es presentar una exposición y análisis comparativo de algunos de los postulados más importantes vinculados al problema de la relación entre derecho y moral, desde lo que actualmente es conocido como antipositivismo y las aportaciones de esta corriente a la teoría jurídica contemporánea, para ello el presente artículo se encuentra dividido en tres apartados, en los dos primeros se presenta una breve exposición de la evolución histórica de las tesis más importantes en torno al ya mencionado problema, en sus dos contextos fundamentales, y en el tercer apartado se lleva a cabo una exposición y análisis de las propuestas de Ronald Dworkin y Robert Alexy.

\section{Contexto iusnaturalista}

En Grecia en el siglo V a. C., Sócrates y los Sofistas iniciaron la controversia entre naturaleza y convención como origen del derecho, el primero derivando las leyes humanas de las leyes de la naturaleza. En la Apología de Sócrates puede observarse que este incluso consideraba la ley divina superior a la creada por los hombres (29d), puede decirse que para Sócrates era una moral universal la que daba fundamento a las leyes creadas por los hombres, pues él concebía a estas como un reflejo de la ley natural, por ese motivo cumple con su sentencia cuando es condenado a muerte, como una forma de adecuar su actuar a esa idea universal de justicia como virtud moral reflejada en las leyes creadas por los hombres. Por su parte los Sofistas postulaban "la idea de una clase de contrato social como fundamento de la ley" (Recaséns, 2003, p.3). El derecho así concebido era creación del hombre gracias a un acuerdo al que llegan los individuos, es decir, concebían como fundamento de las leyes a una especie de moral social (positiva o convencional).

Platón también tuvo una concepción solidaria entre la moral y el derecho, pues para él la ley sería una especie de hilo que va guiando a la ciudad para conducirla de manera correcta, y este hilo provendría de "el razonamiento de algún dios o de algún hombre divino" (Leyes, I, 645b). Por su parte Aristóteles postuló que los principios que le dan 
ENFOQUES JURIDICOS

REVISTA MULTIDISCIPLINAR DEL CEDECS

ISSN 26832070
Artículo: “El problema de la relación entre derecho y moral"

José Luis López Fuentes

Número 03

enero-junio 2021

DOI: https://doi.org/10.25009/ej.v0i3.2563

fundamento al derecho y también a la justicia pueden ser encontrados con ayuda de la razón, en la naturaleza de las cosas, y con ayuda de un criterio teleológico, es decir, que el bien puede ser encontrado en las cosas mismas y también llevó a cabo una diferenciación entre dos clases de justicia, la justicia del juez y la justicia natural como virtud moral, pero consideró que las leyes humanas solamente pueden alcanzar la justica por mero accidente (Ética Nicomáquea, V, 1137a-5), y también que "la gran mayoría de las prescripciones legales se desprenden de la virtud total” (Ética Nicomáquea, V, 1130b-15). En ese sentido, Aristóteles encontraría el fundamento de las leyes en la acepción moral de la virtud.

Por su parte los romanos para referirse a lo que hasta entonces se conocía como derecho natural, usaban distintos términos: ius commune, ius gentium y ius naturale; ese derecho natural era el aplicable a todos los individuos que no eran ciudadanos de Roma, es decir, a los extranjeros, pero los romanos también se valían de este derecho natural como un elemento para interpretar las leyes, es decir, para crear jurisprudencia. Los ciudadanos romanos se regían por el ius civile, y en lo que respecta a las relaciones que surgían entre extranjeros y ciudadanos de Roma se regían por el derecho que era común a todos, es decir, el que proviene de la razón, en ese sentido también se hablaba de derecho natural en cuanto al que regía las relaciones entre particulares (Jus. D. I. I. 4. I.) En ese entendido, puede decirse que los juristas romanos tenían una concepción solidaria de estos derechos, lo que se manifestaba también en una concepción solidaria entre derecho y moral.

Ya en la Edad Media, San Agustín mediante su teoría de la ley eterna formuló un cambio en la concepción que se tenía en el mundo antiguo del iusnaturalismo, pues San Agustín considera un iusnaturalismo basado en la voluntad de Dios, las leyes humanas encontrarían su fundamento en ese mencionado deber moral de obedecer la voluntad de Dios, pero derecho y moral estarían vinculados con ciertos límites, pues para San Agustín la ley positiva solo debía limitarse a prohibir las conductas que no permitan conseguir la paz social (Del Libre Albedrio, V, 1,6,14).

Por su parte Santo Tomás de Aquino también se ocupó de las leyes y de la justicia, distinguiendo entre diversas clases de leyes: ley eterna, ley natural y ley humana, las dos últimas derivadas de la ley eterna, la cual vendría a ser "la razón de la sabiduría divina en cuanto principio directivo de todo acto y todo movimiento" (Suma Teológica, I-II, C. 93 a.1). Santo Tomás también distinguía entre santidad evangélica y justicia derivada del derecho, la primera como virtud moral, y la segunda como justicia jurídica, esto representaría un criterio de distinción entre moral y derecho.

Posteriormente como consecuencia del deseo de desligarse de los planteamientos dominantes en la Edad Media, fundamentalmente teológicos, surgió el iusnaturalismo de corte racionalista, pues "desde fines del siglo XVI hasta finales del XVII la filosofía, y también el derecho buscaron su sitio en el campo de las ciencias exactas." (Bernal, 2016, p.161). Este nuevo iusnaturalismo siguió la tendencia de los postulados imperantes en su época que 
ENFOQUES JURIDICOS

REVISTA MULTIDISCIPLINAR DEL CEDEGS

ISSN 26832070
Artículo: “El problema de la relación entre derecho y moral"

José Luis López Fuentes

Número 03

enero-junio 2021

DOI: https://doi.org/10.25009/ej.v0i3.2563

afirmaban que con solo el uso de la razón podrían desentrañarse las leyes naturales e incluso la naturaleza del hombre. En este contexto Thomas Hobbes creó una nueva teoría sobre el derecho natural, la cual contenía los primeros esbozos del positivismo, pues para Hobbes la autoridad era la fuente de las normas creadas por los hombres "donde no hay poder común, no hay ley" (Leviatán, XIII). Con ello Hobbes estableció una separación entre derecho y moral. Por su parte Jean Jacques Rousseau en el Contrato Social, postuló que la voluntad general era la exclusiva fuente de la ley (Trad. en 2014, pp.29-43), es decir, para Rousseau la ley tenía un origen convencional y encontraría su fundamento en una moral concertada con lo que el derecho y esta clase de moral estarían fuertemente vinculados.

\section{Contexto iuspositivista}

El positivismo jurídico buscó llevar a cabo una aproximación avalorativa y descriptiva del derecho, contrario a las teorías iusnaturalistas. En este contexto, el filósofo y jurista alemán Christian Thomasius postuló una distinción entre normas jurídicas, morales, y reglas sociales, que encontraría como nota característica y fundamental del derecho a la coacción, en ese sentido las normas morales y las reglas sociales al carecer de coacción no podrían ser derecho en un sentido estricto "con ello redujo el derecho al humano positivo" (Hervada, 1996, p.285). Idea precursora de una de las tesis fundamentales del iuspositivismo, la separación entre derecho y moral.

Por otra parte, Immanuel Kant mediante los principios de su ética ofreció importantes concepciones sobre las obligaciones jurídicas y morales. La idea fundamental que se desprende de la teoría ética Kantiana en relación al tema que se viene desarrollando, es que los deberes morales son internos; y los jurídicos externos, en ese sentido, el cumplimiento de los deberes jurídicos solo exige que la acción externa llevada a cabo por las personas esté en conformidad con lo prescrito por el derecho, sin tomar en cuenta cuales fueron los móviles para llevar a cabo esta acción (Trad. en 2008, p.24). Este pensador estableció un criterio fundamental para distinguir entre derecho y moral, que es el móvil para el cumplimiento de las normas, en tanto morales, es el deber el único móvil para su obediencia, y en tanto normas de derecho, se admiten un número más amplio de razones para su observancia. Lo anterior abonó a las teorías positivistas que serían desarrolladas con posterioridad en pro de la separación entre derecho y moral, con ello Kant sentó las bases teóricas del positivismo jurídico.

Con posterioridad Hans Kelsen propuso una tajante separación entre derecho y moral, partiendo de la idea de que un orden jurídico positivo no podía hacer depender su validez de una concepción de justicia absoluta y valida en todo tiempo y lugar (Trad. en 1982, p.79). Con ello Kelsen también postuló la inexistencia de valores absolutos, teniendo en cuenta la gran variedad de concepciones sobre lo que es bueno o justo dadas en las diferentes sociedades y épocas a través de la historia, por lo que el derecho estaría condicionado por 
ENFOQUES JURIDICOS

REVISTA MULTIDISCIPLINAR DEL CEDECS

ISSN 26832070
Artículo: “El problema de la relación entre derecho y moral"

José Luis López Fuentes

Número 03

enero-junio 2021

DOI: https://doi.org/10.25009/ej.v0i3.2563

las variaciones surgidas de la historia y las sociedades; e incluso llegó al extremo de afirmar que el derecho no debía ni siquiera satisfacer un mínimo de moralidad (Trad. en 1982, p.78).

En tiempos más recientes el debate sobre la relación entre derecho y moral ha estado centrado en la posibilidad de una relación conceptual entre estos dos órdenes, ya no en una eventual relación contingente, es decir, sobre si el concepto de derecho incluye necesariamente consideraciones morales. Mediante el análisis de la estructura lógica del derecho se dio un nuevo paso dentro del positivismo jurídico, con la obra de Joseph Raz, el cual, por medio de su positivismo jurídico excluyente defendió una separación conceptual entre derecho y moral, postulando que la existencia y el contenido del derecho dependen exclusivamente de hechos sociales, como actos de legislación, costumbre o precedentes judiciales (Jiménez, 2008, p.196 en nota al pie 21), lo cual indicaría que el derecho es puesto, de que es hecho derecho por la actividad de seres humanos.

Después H. L. A. Hart formularía una tesis desde lo que se conoce como positivismo jurídico incluyente, la cual postula que el derecho y su contenido en determinadas circunstancias se ven condicionados por principios morales (2000, p.51), lo cual abre la posibilidad de una eventual relación entre derecho y moral, mas no una necesaria relación entre ambos sistemas.

\section{Moderna teoría antipositivista}

En los últimos años dos autores fundamentales: Ronald Dworkin y Robert Alexy han ofrecido nuevas e importantes aportaciones a la teoría jurídica y también a la forma de entender la relación entre derecho y moral. Respecto a Dworkin, puede decirse que sus tesis se adscriben a lo que actualmente se conoce como antipositivismo o lo que él ha tenido a bien llamar interpretativismo, dicha teoría postula que el derecho no solo está integrado por reglas específicas promulgadas de conformidad con las prácticas aceptadas de la comunidad, sino también de los principios que aportan a dichas reglas la mejor justificación moral (2014, pp.485-486). Dworkin también dedicó parte de su obra a llevar a cabo una crítica del positivismo como teoría sobre el derecho, principalmente a las tesis elaboradas por H. L. A. Hart con el que mantuvo diversos debates teóricos vinculados con sus concepciones sobre los fundamentos últimos del derecho y su manera de entender al mismo. Por otro lado, las tesis elaboradas por Robert Alexy parten desde la idea de la pretensión de corrección como elemento necesario del derecho. Por razones de espacio la única intención de este apartado es brindarle al lector una perspectiva general de las principales ideas y postulados que los mencionados autores han brindado en torno al debate contemporáneo sobre el problema de la relación entre derecho y moral; y sus aportaciones a la teoría jurídica contemporánea, que se adscriben a lo que hoy es conocido como antipositivismo.

Los postulados de Ronald Dworkin se caracterizan por brindarle una especial relevancia a la moral y a la política para la explicación del derecho, contrario a la corriente positivista 
ENFOQUES JURIDICOS

REVISTA MULTIDISCIPLINAR DEL CEDECS

ISSN 26832070
Artículo: “El problema de la relación entre derecho y moral"

José Luis López Fuentes

Número 03

enero-junio 2021

DOI: https://doi.org/10.25009/ej.v0i3.2563

más pura, que pretende dejar de lado a la moral y a cualquier clase de compromiso político, dada su manera de hacer teoría sobre el derecho. Las críticas de Dworkin al positivismo jurídico estuvieron especialmente dirigidas a la versión del positivismo jurídico elaborada por de H. L. A. Hart, como lo señalaba en Los Derechos en Serio (1989, p.72). Otra característica de las obras elaboradas por Dworkin, es que tratan de brindar una profunda explicación sobre la manera en que los jueces interpretan y aplican la ley, cuestión que abordó en El imperio de la justicia.

Las teorías de Ronald Dworkin se ubican en el sistema jurídico de Estados Unidos de América, razón por la cual ha sido blanco de múltiples críticas, pues sus detractores consideraron esto un sesgo, si bien es cierto que él se sirve mayormente de determinados casos famosos dentro del sistema legal estadounidense y los utiliza como ejemplos para explicar sus postulados, Dworkin afirma que sus tesis bien pueden servir de referencia para explicar diversos sistemas jurídicos "la mejor respuesta a la cuestión de si mi teoría del derecho pretende ser universal o parroquial es: ambas cosas" (2007, p.252). Dworkin concibe una teoría liberal que pone particular atención a los derechos individuales y les otorga una especial jerarquía, incluso por encima de beneficios colectivos, es por ello que su pensamiento se aleja de teorías como la utilitarista (1989), e intenta dar razones para la justificación de la relación existente entre derecho, moral y política.

Dworkin comienza su crítica al positivismo, precisando lo que considera son las tres notas características de esta corriente de pensamiento jurídico, la primera es que el positivismo jurídico concibe al derecho como un conjunto de normas destinadas a establecer qué comportamientos serán sancionados, los cuales pueden ser identificadas mediante determinados criterios de origen. La segunda, es que las mencionadas normas constituyen todo lo que el derecho es, y en caso de que surgiera una situación no prevista en las mencionadas normas, los jueces usarían su discrecionalidad para resolverla. La tercera característica se refiera a que, en las mencionadas situaciones no previstas, cuando un juez resuelve haciendo uso de su discreción, es decir, cuando no existe una norma jurídica valida aplicable al caso, que imponga una obligación o prohibición, se está yendo más allá del derecho, por lo que no puede afirmarse que existe una obligación jurídica. (1989, pp.6566). Con las tres características del positivismo jurídico expuestas por Dworkin, es posible observar que una de las cuestiones que más polémica suscita es el relacionado con la discrecionalidad de los jueces, y saber de qué criterios se sirven para resolver casos difíciles, en los que no existe una norma adecuada aplicable. En relación a este tema, Hart afirmaría en su libro Post scríptum al concepto del derecho, que el derecho es incompleto porque en todos los órdenes jurídicos siempre surgen determinados casos que no se ajustan a las normas jurídicas existentes, y por consiguiente el derecho no puede dar una solución específica. En ese sentido, lo que Hart sugiere es que el juez debe llevar a cabo una labor de creación, aunque limitada por el derecho ya existente (2000, p.54). 
ENFOQUES JURIDICOS

REVISTA MULTIDISCIPLINAR DEL CEDECS

ISSN 26832070
Artículo: “El problema de la relación entre derecho y moral"

José Luis López Fuentes

Número 03

enero-junio 2021

DOI: https://doi.org/10.25009/ej.v0i3.2563

Dworkin sostiene que dicha teoría es errónea, ya que existen otros criterios que entran en juego cuando los tribunales toman sus decisiones, a estos criterios los denomina principios, los cuales son patrones que constituyen una exigencia de justicia, equidad, o de cualquier otra dimensión de la moralidad (1989, p.72). Al respecto Dworkin afirma que en el momento que los jueces deciden un proceso, no solo toman en consideración a normas jurídicas, sino también a los menciona dos principios, como por ejemplo el principio de que nadie puede beneficiarse de su propio delito (1989:102). Para ejemplificar la relevancia de los principios, Dworkin cita un caso en el cual un hombre asesinó a su abuelo envenenándolo, en lo que fue conocido como El caso Elmer, dicho hombre era el heredero de la mayor parte de los bienes de su abuelo. Al parecer Elmer decidió llevar a cabo el asesinato porque su abuelo contrajo nuevas nupcias, lo que ponía en riesgo su estatus de principal heredero. Teniendo en cuenta lo sucedido surgió una interrogante, el cual era saber si Elmer tenía derecho a reclamar dicha herencia a pesar de haber asesinado a su abuelo para obtenerla, pues las hijas de este último creían que al Elmer haber cometido el asesinato, no tenía derecho a reclamar la parte de la herencia que se estipulaba en el testamento; y que por lo tanto esa parte les correspondía a ellas. El abogado de Elmer fundamentó la defensa en que el testamento no vulneraba ninguna parte del estatuto de testamentos vigente, y que por lo tanto el mismo era válido, afirmando que, si los jueces fallaban a favor de las hijas del fallecido, se estaría sustituyendo la ley por sus convicciones morales (Dworkin, 2012, p.25). En un primer momento los jueces del caso creyeron que su fallo debía estar de acuerdo con la letra de la ley, lo que ciertamente puede considerarse una interpretación literal y mecánica, pero hay ciertos aspectos que debemos considerar en esta forma de interpretación que pretendían adoptar en un inicio los jueces de este caso, y por uno de ellos en particular, el juez Gray, quien votó a favor de que le fuera entregada la parte de la herencia que le correspondía a Elmer, aduciendo que si este perdía dicha herencia, ello constituiría un castigo más para él, aparte de la condena que debía cumplir en prisión, por lo que era necesario tener en cuenta el principio de que nadie puede recibir un castigo mayor al establecido con anterioridad para un determinado delito (Dworkin, 2012, pp.26-27).

Otro de los jueces, el juez Earl, utilizó una forma distinta de interpretación, pues postulaba que los jueces debían considerar el hecho de que el estatuto de testamentos vigente formaba parte de un sistema mayor, es decir el derecho, y que este, en otra parte, respetaba determinados principios de justicia, teniendo esto en cuenta, resulta coherente pensar que cuando se legisla se pretende respetar dichos principios, a menos de que se manifieste expresamente lo contrario, por lo que, si el estatuto de testamentos vigente en ese entonces formaba parte de un sistema mayor conocido como derecho, debía respetar los mismos principios de justicia, para que dicho sistema entendido como un todo fuera coherente. Esta forma de interpretación fue la vencedora al obtener el voto de la mayoría 
ENFOQUES JURIDICOS

REVISTA MULTIDISCIPLINAR DEL CEDECS

ISSN 26832070
Artículo: “El problema de la relación entre derecho y moral"

José Luis López Fuentes

Número 03

enero-junio 2021

DOI: https://doi.org/10.25009/ej.v0i3.2563

de los jueces, por lo que Elmer perdió su parte de la herencia, debido a que el juez Earl defendió que "la ley en otra parte respeta el principio de que nadie debe aprovecharse de sus propios errores, de modo que el estatuto de testamentos tendría que leerse para negar la herencia a alguien que ha matado para obtenerla" (Dworkin, 2012, p.27).

Según la teoría de Dwokin, los señalados principios y su relevancia en la decisión de casos difíciles sirven para refutar la tesis de la regla de reconocimiento elaborada por Hart, pues sabemos que este último autor postula que dicha regla de reconocimiento proporciona criterios formales de validez que permiten identificar al derecho y diferenciarlo de otros sistemas que regulan la conducta de las personas, como la moral (1998, pp.125-137), pero al incluir a los principios en la aplicación de la ley, como Dworkin afirma que sucede en casos complejos, la regla de reconocimiento no serviría para identificar a dichos principios, pues ellos se refieren a contenidos morales, más no formales. Como defensa a la crítica anteriormente señalada, Hart declararía que "la regla de reconocimiento puede proporcionar pruebas referidas no solo al contenido fáctico de las normas sino a su conformidad con valores o principios morales sustantivos" (2000, p.37). Aunque para Hart la relación entre derecho y moral es meramente contingente; es decir, no necesaria, pero como consecuencia de que aceptara que en ciertos casos la regla de reconocimiento incluye criterios de moralidad, Dworkin afirmaría que en los países con sistemas jurídicos complejos “... no se puede encontrar semejante criterio fundamental, y que en tales países no es posible establecer una distinción final entre los estándares legales y morales" $(1989$, p.102). Con ello podemos ver que para Dworkin la regla de reconocimiento como es concebida por Hart no sirve para identificar a todo el derecho y sus normas, ni tampoco para diferenciar a estas últimas de otra clase de normas, como las morales, es por ello que para Dworkin no existe una separación entre derecho y moral.

Respecto a Robert Alexy puede decirse que sus ideas sobre el derecho abogan por una relación conceptual necesaria entre el derecho y la moral, lo que parte de dos argumentos fundamentales: argumento de la injusticia y argumento de la corrección. El primero se refiere a que, si una norma jurídica excede un cierto grado de injusticia, dejaría de ser una norma jurídica, pues según Alexy (2008) “los defectos morales socavan la validez jurídica si y sólo si se traspasa el umbral de la injusticia extrema. Por debajo de ese umbral, los efectos de los defectos morales se confinan a atribuir al derecho un carácter defectuoso" ( pp.8283). En ese sentido, para Alexy el grado de injusticia contenido en una norma, funciona como un criterio clasificatorio de la misma, es decir, es un criterio para clasificar una norma como jurídica o no jurídica. El segundo argumento de Alexy en favor de una relación conceptual entre derecho y moral, es el argumento de la corrección; el cual consiste en que "las normas jurídicas individuales y las decisiones judiciales, así como el sistema jurídico en su totalidad, necesariamente tiene una pretensión de corrección" (1993, p.51), o, en otras

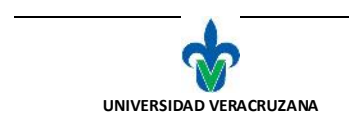

pp. $65-80$

드로. 
ENFOQUES JURIDICOS

REVISTA MULTIDISCIPLINAR DEL CEDECS

ISSN 26832070
Artículo: “El problema de la relación entre derecho y moral"

José Luis López Fuentes

Número 03

enero-junio 2021

DOI: https://doi.org/10.25009/ej.v0i3.2563

palabras, cuando se crea o aplica el derecho, hay necesariamente en los participantes una pretensión de corrección moral (Borda, 2001, p.19).

Analizando más detalladamente el argumento fuerte de Alexy en favor de una relación conceptual y necesaria entre derecho y moral, el argumento de la injusticia, hay que tener en cuenta que este puede referirse a normas jurídicas aisladas o al sistema jurídico en su totalidad (Alexy, 1993, p.45). En relación a normas individuales, el argumento de la injusticia de Alexy bebe en gran medida de la fórmula elaborada por Radbruch; que puede ser resumida en la idea de que la injusticia extrema no es derecho. Con ello no se está afirmando que una norma jurídica al ser injusta, pierde por esa razón su calidad de jurídica; sino que esto ocurriría si una norma sobrepasa el umbral de la injustica extrema. Para ejemplificar la idea anterior Alexy utiliza algunos ejemplos relacionados con determinadas regulaciones en la legislación alemana, acaecidas durante la Segunda Guerra Mundial, en los cuales se revocaba la ciudadanía alemana a emigrantes judíos, y plantea un caso hipotético en el cual un jurista extranjero desea escribir un artículo sobre el sistema legal alemán nazi; en relación a los judíos que perdieron la ciudadanía alemana debido a su condición racial, por lo que debe plantearse la siguiente cuestión "A es expatriado correctamente de acuerdo con el criterio de validez en Alemania, y su expatriación es socialmente efectiva. Pero, ¿es esto derecho?" (1993, p.47). Para dar respuesta a esta última interrogante lo fundamental sería preguntarse si el caso de los judíos expatriados sobrepasa un grado de injustica extrema, únicamente en el supuesto de que el lector aceptara como verdadera la fórmula de Radbruch, pero ello aun dejaría una cuestión abierta, el saber si incluso la injusticia extrema puede ser derecho, Alexy llega a la conclusión de que el argumento de la injusticia en relación a normas individuales "no exige ningún tipo de compatibilidad plena entre el derecho y la moral" (2008, p.70). Pues que si una norma es injusta (no en extremo) puede ser considerada jurídica.

Lo anterior no constituye el final del argumento de la injusticia en la tesis de Alexy, debido a que en este punto el análisis llevado a cabo por él es enfocado en un sentido distinto, para saber si el sistema jurídico entendido como totalidad tiene una relación conceptual y necesaria con la moral. En ese entendido, Alexy habla de dos tipos de requerimientos para poder abogar en favor de esta relación, requerimientos formales $y$ materiales, Los primeros se refieren a cuestiones como la generalidad de las leyes y la irretroactividad de las mismas, en tanto que los segundos se relacionan con la satisfacción de criterios de justicia, como no matar o no robar. (1993, p.48). En su intento por demostrar una conexión conceptual entre derecho y moral, Alexy (1993) precisa que "hay dos tipos de ordenes sociales que no pueden ser sistemas jurídicos por razones conceptuales, sin importar si tienen, o no, validez continua: el orden sin sentido y el orden predatorio" ( $\mathrm{p} .49$ ). Para explicar estas dos clases de órdenes utiliza como ejemplo a un grupo de bandidos que gobiernan a una amplia cantidad de individuos. El orden sin sentido surge cuando 
ENFOQUES JURIDICOS

REVISTA MULTIDISCIPLINAR DEL CEDECS

ISSN 26832070
Artículo: “El problema de la relación entre derecho y moral"

José Luis López Fuentes

Número 03

enero-junio 2021

DOI: https://doi.org/10.25009/ej.v0i3.2563

determinados sujetos gobiernan sin revelar sus intenciones o quien es el creador de las reglas del grupo, y además no se persiguen los intereses de los gobernados. Este último orden se transforma en predatorio cuando los bandidos se organizan y establecen una jerarquía entre ellos, a la vez que se prohíbe la violencia. Los dos tipos de ordenes recientemente mencionados, por razones conceptuales no pueden ser considerados como jurídicos en la tesis de Alexy, la pregunta que surge de inmediatos es ¿Cuál es el elemento faltante para que tales ordenes puedan ser concebidos como jurídicos? Este elemento surgiría cuando los bandidos se convirtieran en legisladores, con la intención de seguir explotando a los gobernados, pero ahora sirviéndose de un sistema de reglas. Al respecto Alexy manifiesta que en este caso los bandidos "Continúan explotando a los súbditos. Pero ahora llevan a cabo la explotación por medio de actividades guiadas por reglas, afirman que tal práctica es correcta, porque sirve a algún propósito superior, como, por ejemplo, el desarrollo del pueblo" (1993, p.50). Aunque un sistema como el del último ejemplo pueda ser percibido como injusto, para Alexy ya no resulta inviable considerarlo conceptualmente jurídico, pues en él ya se encuentra el elemento que le faltaba al orden predatorio, y que es; la pretensión de corrección, pues todo orden positivo, tiene la pretensión de ser justo, por esta razón Alexy afirma que la pretensión de corrección es un elemento necesario y que se encuentra intrínsecamente ligado al concepto de derecho, lo que viene a representar el nexo entre moral y derecho.

Según Alexy las normas jurídicas aisladas, las decisiones judiciales y el sistema jurídico entendido como un todo, tienen de manera necesaria una pretensión de corrección, pero qué significa en la tesis de Alexy el enunciado "tienen una pretensión". Al respecto él puntualiza lo siguiente: "Que el "derecho» eleva una pretensión significa que lo hacen las personas que ejercen una u otra competencia jurídica" (2008, p.63). Debemos tener en cuenta la diferencia que existe entre realizar y satisfacer; pues decir que una ley formula una pretensión de corrección, no significa que efectivamente la satisfaga, lo mismo ocurre con un sistema jurídico entendido como un todo, para Alexy esta es una importante diferenciación que debemos tener presente para entender mejor su tesis de la pretensión de corrección. A partir de dicha diferenciación podemos establecer la clasificación que se presenta en la siguiente tabla.

Tabla 1. Tesis de la pretensión de corrección de Robert Alexy

\begin{tabular}{|l|l|l|}
\hline Relación entre derecho y moral & Formulación de pretensión & Satisfacción de la pretensión \\
\hline Sistemas jurídicos & Clasificatoria & Cualificatoria \\
\hline Normas jurídicas & Cualificatoria & Cualificatoria \\
\hline
\end{tabular}

Elaboración propia, con base en Borda Villar, Luis, Serie de teoría jurídica y filosofía del derecho No. 18. Bogotá, Colombia, 2001, Universidad Externado de Colombia, p. 20. 
ENFOQUES JURIDICOS

REVISTA MULTIDISCIPLINAR DEL CEDECS

ISSN 26832070
Artículo: “El problema de la relación entre derecho y moral"

José Luis López Fuentes

Número 03

enero-junio 2021

DOI: https://doi.org/10.25009/ej.v0i3.2563

Lo que la tabla anterior muestra es que un sistema jurídico entendido como totalidad que no lleve a cabo la formulación de una pretensión de corrección no será considerado un sistema jurídico, es decir, que dicha pretensión de corrección, en relación con los sistemas normativos entendidos como un todo, constituye un criterio clasificatorio. Por otro lado, si un sistema jurídico formula la pretensión de corrección, pero no llega a satisfacerla, es un sistema defectuoso (Alexy, 1993, p.51). Por ello es que en este caso se considera a la pretensión de corrección, como un criterio cualificatorio de dicho sistema. Si hablamos de normas legales aisladas, podemos decir que, si estas no formulan una pretensión de corrección, siguen siendo consideradas como normas legales, de la misma manera que si formulan dicha pretensión, pero no llegan a satisfacerla, pues en ambos casos no perderán su carácter de normas jurídicas, simplemente serán consideradas normas legales deficientes, lo mismo opera para las decisiones judiciales.

Alexy nos dice que su teoría puede ser combatida por dos caminos, el primero sería rechazar la conexión conceptual entre la pretensión de corrección y el derecho, y el segundo, sería afirmar que, si bien dicha pretensión de corrección está conceptualmente unida al derecho, esto resulta irrelevante y que no incluye cuestiones morales (1993, p.52). Para rebatir el primer argumento en contra de su tesis, Alexy utiliza un ejemplo hipotético en el cual una minoría domina a una gran parte de la población en un Estado X; y de ello obtienen grandes beneficios que, por supuesto quieren seguir conservando, pero al mismo tiempo desean ser honestos; el siguiente es el primer artículo de la Carta Magna del supuesto Estado: X es una república, soberana, federal e injusta. Este artículo constitucional adolece de lo que Alexy llama un defecto conceptual, pues en sus palabras "una pretensión de corrección se relaciona necesariamente con el acto de establecer una constitución, y en este caso se trata, principalmente, de una pretensión de justicia" (1993, p.53). Es decir, que en el citado ejemplo; al negar en el primer artículo de la Constitución la pretensión de justicia se está llevando a cabo una contradicción performativa. El autor en comento brinda otro ejemplo más al respecto de esta clase de contradicciones performativas, el cual consiste en que un determinado juez emite una resolución en el siguiente sentido: el acusado es -equivocadamente- sentenciado a cadena perpetua. En primer lugar, debemos tener en cuenta el sentido en que se utiliza la palabra "equivocadamente" en el ejemplo; pues la misma puede ser susceptible de diversas interpretaciones, Alexy precisa que, en el citado ejemplo, dicho término se refiere a que el derecho ha sido interpretado de manera incorrecta. Una vez precisado lo anterior, es posible entender la postura de Alexy cuando este afirma que una decisión judicial busca siempre aplicar de manera correcta el derecho, en ese sentido "El contenido del veredicto contradice la pretensión hecha por el acto institucional de enunciar la sentencia" (1993, p.54). Estos dos ejemplos según la tesis de Alexy permiten poner de manifiesto que los participantes en un sistema legal, es decir, los individuos que crean y aplican el derecho tienen indudablemente la pretensión de 
ENFOQUES JURIDICOS

REVISTA MULTIDISCIPLINAR DEL CEDECS

ISSN 26832070
Artículo: “El problema de la relación entre derecho y moral"

José Luis López Fuentes

Número 03

enero-junio 2021

DOI: https://doi.org/10.25009/ej.v0i3.2563

corrección, aunque para él esto aún no es suficiente para fundamentar una relación conceptual y necesaria entre derecho y moral, por lo que Alexy se apoya también en la teoría del discurso y en la del caso especial para lograr dicho cometido.

Para el autor que se aborda un sistema jurídico no puede descansar únicamente en el poder. Aunado a lo anterior, para la teoría de Alexy es fundamental tener en cuenta dos cuestiones, la primera se refiere a que el derecho es incompleto como lo postulaba Hart, y que los casos no previstos, es decir, los casos difíciles, no pueden ser resueltos echando mano exclusivamente de razones que deriven del derecho positivo $(2008$, p.67). Lo que a su vez conduciría a dos vías para resolver esta clase de casos difíciles, la primera es no basarse en razón alguna, y la segunda se refiere a resolver dichos casos difíciles con razones ajenas al sistema jurídico. Dada la naturaleza parcialmente indeterminada o incompleta del derecho, Alexy llega a la conclusión de que los operadores jurídicos para resolver los mencionados casos difíciles, deben echar mano de criterios que no pertenecen propiamente al ámbito de lo jurídico, en ese sentido, se vuelve necesario considerar las razones morales, debido a que:

Cuando las razones del derecho positivo se agotan la pretensión de corrección permite recurrir a razones de toda laya, pero cuando hay buenas razones para adoptar una decisión judicial, se otorga prioridad a las consideraciones de justicia sobre todas las demás consideraciones que no están basadas en el derecho positivo (Alexy, 2008, pp.67-68).

Es por ello que Robert Alexy encuentra una relación necesaria entre derecho y moral. Aunque dicha pretensión de corrección en relación con las normas jurídicas individuales, solo sirve como criterio calificativo, es decir, para catalogar a una norma legal como defectuosa o no, cuestión de la que Alexy es plenamente consciente, pero a pesar de ello afirma que esta es una situación que no pierde importancia debido a que "la conexión calificadora significa que las decisiones jurídicas moralmente erróneas son, de forma necesaria, jurídicamente erróneas" (2008, p.68).

\section{Consideraciones finales}

Parece ser que el debate entre el positivismo y el no-positivismo está centrado fundamentalmente en la relación entre derecho y moral, sin duda las aportaciones de Ronald Dworkin y Robert Alexy a la forma de entender esta relación han sido innovadoras y polémicas en la teoría jurídica contemporánea. Aportaciones que intentaron dar una explicación del derecho alejada del positivismo, que postulan la utilización de principios con contenido moral, y su relevancia en la solución de casos difíciles, lo que a su vez sostiene que el razonamiento moral está intrínsecamente ligado al razonamiento legal, teniendo en cuenta que dichos principios se encuentran vinculados a una dimensión de la moralidad. Lo anterior hoy en día tiene una gran relevancia en la explicación de la estructura y funcionamiento de determinados sistemas jurídicos, sobre todo en los modernos Estados constitucionales de derecho, en los que puede decirse que la Constitución modela un orden 
ENFOQUES JURIDICOS

REVISTA MULTIDISCIPLINAR DEL CEDECS

ISSN 26832070

Número 03

enero-junio 2021
Artículo: “El problema de la relación entre derecho y moral"

José Luis López Fuentes

DOI: https://doi.org/10.25009/ej.v0i3.2563

objetivo de valores, en ese sentido, resultan sumamente relevantes las aportaciones de Dworkin, pues mediante sus postulados buscó fundamentar una teoría que brindara una relevancia especial a la protección de los derechos individuales, lo cual parte desde la idea de que los jueces deben resguardar dicho orden objetivo de valores. Alexy por su parte al abogar por una relación conceptual y necesaria entre derecho y moral, manifestó que el derecho no solo tiene una dimensión real o fáctica, sino también una dimensión ideal o crítica. Esta última dimensión, es la que se encontraría intrínsecamente relacionada con la pretensión de corrección, que según Alexy es una pretensión de corrección moral.

Sin duda el positivismo jurídico ha brindado grandes aportaciones a nuestra comprensión sobre el derecho, y si bien grandes pensadores positivistas como Hans Kelsen y Joseph Raz llegaron a postular una separación absoluta entre derecho y moral, actualmente los principios previstos en las Constituciones de los Estados y en las modernas cartas internacionales de derechos humanos, como el principio de dignidad humana, libertad e igualdad, tienen un papel muy relevante en la interpretación y aplicación de la ley, parece ser que el mundo de los valores aún tiene mucho que aportar al pensamiento jurídico, como lo ha sugerido el moderno antipositivismo. 
ENFOQUES JURIDICOS

REVISTA MULTIDISCIPLINAR DEL CEDECS

ISSN 26832070

Número 03

enero-junio 2021
Artículo: "El problema de la relación entre derecho y moral"

José Luis López Fuentes

DOI:

\section{Fuentes de consulta}

Alexy, R. (1993). Derecho y Razón Práctica. México: Fontamara.

Alexy, R. (2008). El concepto y la naturaleza del derecho. Trad. Carlos Bernal Pulido. MadridBarcelona-Buenos Aires: Marcial Pons.

Aristóteles. (1985). Ética Nicomáquea. Ética Eudemia. Trad. Julio Pallí Bonet, Madrid, España: Gredos.

Bernal, B. (2016). Historia del derecho. México: IIJ-UNAM. Recuperado de: https://biblio.juridicas.unam.mx/bjv/detalle-libro/3263-historia-del-derechocoleccion-cultura-juridica

Borda, L. (2001). Serie de teoría jurídica y filosofía del derecho No. 18. Bogotá, Colombia: Universidad Externado de Colombia.

Dworkin, R. (2012). El imperio de la justicia. De la teoría general del derecho e interpretación de los jueces y de la integridad política y legal como clave de la teoría y práctica. Trad. Claudia Ferrari. España: Gedisa.

Dworkin, R. (2014). Justicia para Erizos. Trad. Horacio Pons. México: Fondo de Cultura Económica.

Dworkin, R. (2007). La justicia con toga. Trad. Marisa Iglesias Villa e Iñigo Ortiz De Urbina Jimeno. Madrid-Barcelona-Buenos Aires: Marcial Pons.

Dworkin, R. (1989). Los Derechos en Serio. Trad. Marta Guastavino. Barcelona, España: Ariel.

Hart, H. L. A. (1998). El concepto de derecho. Trad. Genaro R. Carrió. Buenos Aires, Argentina: Abeledo-Perrot. 
ENFOQUES JURÍDICOS

REVISTA MULTIDISCIPLINAR DEL CEDECS

ISSN 26832070

Número 03

enero-junio 2021
Artículo: "El problema de la relación entre derecho y moral"

José Luis López Fuentes

DOI:

Hart, H. L. A. (2000). Post scríptum al concepto del derecho. Trad. Rolando Tamayo y Salmorán. México: IIJ-UNAM. Recuperado de: https://biblio.juridicas.unam.mx/bjv/detalle-libro/38-post-scriptum-al-conceptodel-derecho

Hervada, J. (1996). Historia de la ciencia del derecho natural. Tercera edición. España: Eunsa.

Hobbes, T. (1980). Leviatán. Segunda edición. Trad. Antonio Escohotado. Madrid, España: Editora Nacional.

Jiménez, R. M. (2008). Una Metateoría del Positivismo Jurídico. Madrid, España: Marcial Pons.

Justiniano. (1889). Cuerpo del derecho civil romano t. I Instituta-Digesto. Trad. Ildefonso L. García del Corral. Barcelona: IIJ-UNAM. Recuperado de: https://biblio.juridicas.unam.mx/bjv/detalle-libro/600-cuerpo-del-derecho-civilromano-t-i-instituta-digesto

Kant, I. (2008). La Metafísica de las Costumbres. Trad. Adela Cortina Orts y Jesus Conill Sancho. España: Tecnos.

Kelsen, H. (1982). Teoría pura del derecho. Segunda edición. Trad. Roberto J. Vernengo. México: IIJ-UNAM. Recuperado de: https://biblio.juridicas.unam.mx/bjv/detallelibro/1039-teoria-pura-del-derecho-2a-ed

Platón. (1981). Diálogos. Trad. J. Calonge Ruiz, E. Lledó Iñigo y C. García Gual. Madrid, España: Gredos.

Platón. (1999). Diálogos Vol. 8 Leyes (Libros I-VI). Trad. Francisco Lisi. Madrid, España: Gredos.

Recaséns, L. (2003). Historia de las doctrinas sobre el contrato social. México: IIJ-UNAM. Recuperado de: https://biblio.juridicas.unam.mx/bjv/detalle-libro/4142-historiade-las-doctrinas-sobre-el-contrato-social-coleccion-facultad-de-derecho

Rousseau, J. J. (2014). El contrato social. Trad. Enrique López Castellón. Madrid, España: Edimat 
ENFOQUES JURIDICOS

REVISTA MUITIDISCIPLINAR DEL CEDECS

ISSN 26832070

Número 03

enero-junio 2021
Artículo: "El problema de la relación entre derecho y moral"

José Luis López Fuentes

DOI:

San Agustín. (1963). Del libre Albedrio, en Obras de San Agustín III. Madrid, España: Biblioteca de Autores Cristianos.

Santo Tomás de Aquino. (1993). Suma de Teología II, parte I-Il. Madrid, España: Biblioteca de Autores Cristianos. 